\title{
UNINTENDED CONSEQUENCES: HOW QUALIFICATION CONSTRAINS INNOVATION
}

\author{
Craig A. Brice \\ National Aeronautics and Space Administration \\ Langley Research Center, Hampton, VA, 23681 USA
}

\begin{abstract}
The development and implementation of new materials and manufacturing processes for aerospace application is often hindered by the high cost and long time span associated with current qualification procedures. The data requirements necessary for material and process qualification are extensive and often require millions of dollars and multiple years to complete. Furthermore, these qualification data can become obsolete for even minor changes to the processing route. This burden is a serious impediment to the pursuit of revolutionary new materials and more affordable processing methods for air vehicle structures. The application of integrated computational materials engineering methods to this problem can help to reduce the barriers to rapid insertion of new materials and processes. By establishing predictive capability for the development of microstructural features in relation to processing and relating this to critical property characteristics, a streamlined approach to qualification is possible. This paper critically examines the advantages and challenges to a modeling-assisted qualification approach for aerospace structural materials. An example of how this approach might apply towards the emerging field of additive manufacturing is discussed in detail.
\end{abstract}

\section{Introduction}

For aerospace metallic materials, the qualification procedure for the introduction of new alloys is very rigorous and well defined [1, 2]. Emphasis is placed on thorough statistical determination of minimum properties with a very high degree of confidence. The statistical basis for these minimum design values are governed by the criticality of the structure. Critical, non-redundant structure usually requires A-Basis minimum values while critical, redundant structure requires BBasis values. The significance of these classifications with regard to qualification lies in the data requirements necessary to satisfy the statistical methods used. For A-Basis qualification, in a material that cannot be described by a parametric distribution (e.g. Weibull), the minimum data requirement for directly determined properties is 299 samples for an isotropic material [3]. Anisotropy in the material may require additional testing in multiple orientations. Thus, the determination of A-Basis minimum design values for tensile yield and ultimate strength in an orthotropic material requires a minimum of 897 tests. The testing required to fully define the mechanical behavior of the material from static and dynamic perspectives often requires many thousands of individual tests.

This level of rigorous material characterization has certainly contributed to the fact that catastrophic structural failures in both civilian and military aircraft are exceedingly rare. The consequence of this rigor, however, is an exceptionally high cost and time span barrier to the introduction of new materials. The cost and time for such an effort can often exceed millions of 
dollars and require five to fifteen years to complete. As we enter a new era of computationally driven materials design, this qualification barrier will likely constrain the pace of innovation and hinder progress. For computationally driven alloy design to effectively work in the aerospace market, a new qualification paradigm is necessary.

\section{Historical Perspective}

\section{Discussion}

The roots of innovation in many high technology areas can be traced to geopolitical events that have demanded creativity and rapid advancement of new ideas [4]. The path to innovation for advanced metallic materials is no exception. For most of the $20^{\text {th }}$ century the pursuit of military and space dominance created an aggressive, risk-tolerant environment which led to the development of many new alloys such as $\mathrm{Al} 2219$ and Ti-6Al-4V that are still in wide use today. Unfortunately, the ebb and flow of these driving forces for innovation limits the resources available (both time and money) for sustained progress. The shift away from empirically developed materials will require a much deeper understanding of process-microstructureproperty relationships. This can be realized through computationally driven alloy design.

Recognizing and addressing up-front the constraints imposed on the materials development community, under the current empirical qualification methodology, is an important step and must not be ignored. The production rate constraints of the conventional processing methods (e.g. forging) can introduce multiple-year lead times. Often this means that the long lead time items such as landing gear, wing carry-through bulkhead, etc. need to be ordered prior to finalizing the design of the vehicle. The legacy method for qualifying metallic materials has left the material developers and the structures designers out of sync. The time required to fully characterize a promising new material often exceeds the window of time available to make design decisions. Shortening, and perhaps eliminating, this timing disconnect is essential in order to take full advantage of what predictive modeling can offer. Much in the same way a structures designer uses finite element analysis to optimize the structural configuration of a part, the materials designer will be able to conduct similar optimization of the material in "real time".

This perspective on the disconnect between the materials designers and the structural integrators is not new [5]. A large program funded by the Defense Advanced Research Projects Agency in the early 2000's addressed this very issue. Under the Accelerated Insertion of Materials (AIM) program, a concerted effort was made to address the broad challenges involved in introducing new materials into various markets [6]. The goals and objectives of this program are still relevant today even though the project is complete. It is worth reconsidering the AIM strategy in light of an increasing focus on computational materials design for additive manufacturing techniques.

\section{Additive Manufacturing - a Qualification Constrained Process}

Affordability demands are beginning to reshape the manufacturing landscape within the aerospace sector. Even in defense programs, where performance demands can quickly balloon costs, affordability is becoming a key metric. This is evident on the Lockheed Martin F-35 program where a pilot scale implementation of additive manufacturing is currently underway as an affordability initiative [7]. In this case, large-scale electron beam wire additive manufacturing using Ti-6Al-4V alloy is being used as a direct replacement for forged structures of the same 
alloy. While the material has not changed, the fabrication process has; based on current methodology this requires requalification of the material. Given the overall magnitude of the F35 program and the corresponding total accumulated cost savings for a relatively high production run platform, the expense of A/B-Basis re-qualification can be justified. This is not the case for the vast majority of other candidate vehicles.

The data generated under the qualification program effectively "fixes" the materials and procedures in-place and requires the process to become static. While this is desirable and necessary for a standardized and repeatable process, it also limits the ability to seek improvements in the process (and in the materials generated by the process). The additive manufacturing approach allows for more degrees of freedom in the fabrication process. Multiple process paths can yield the acceptable end product, both microstructurally and mechanically. Furthermore, the conditions and/or material chosen in the qualification study may turn out to not be the ideal path as the process evolves and matures. Unfortunately, any excursion from the standard deposition process, as established in the specification procedures, will not be allowed under the current methodology.

The challenge for the additive manufacturing community is that the process segment of the process-microstructure-property relationship is not necessarily uniform or static. This implies the need for an outcome-based approach for material qualification. Currently, design minimum values are linked to a specific product form and are often further segmented based on section thickness. All of this is directly related to the microstructure (and indirectly to the resultant properties) though microstructure is not a governing criterion in the specification itself. Put another way, if two wildly different processing routes for the same material produce identical microstructures, the current methodology treats them as two different materials. The focus clearly needs to be on the outcome of the process, not the process itself. The complication lies in the fact that it will no doubt be contentious proving two microstructures are "identical". This is where computational methods can help by filling in the "continuum" in the processmicrostructure-property relationship where data does not exist to predict subtle variations in the outcome of the process.

Sharing the Qualification Burden

Historically, the qualification burden for a new material has been the responsibility of the primary producer. For aerospace metallic materials, the majority of these producers are large semi-integrated operations that have the financial resources to undertake an expensive qualification program for a promising new material. Much like the case for additive manufacturing on F-35, the magnitude of the qualification effort requires large companies that can allocate the financial resources. The continued advancement of additive manufacturing will require a more nimble, less cost-intensive approach. For with these new methods, the responsibility for final melting will now lie with much smaller companies that can not afford a large qualification campaign. An effective way for these small producers to work together towards qualification is necessary.

A novel approach taken by the fiber composite community is to pool resources and share data through a non-competitive organization. This method was established through the Advanced General Aviation Technology Experiments (AGATE) Consortium and managed through the 
National Institute for Aviation Research at Wichita State University [8]. There are a number of advantages to a centralized non-proprietary repository of certified test data. First, an equivalency method for qualification can be utilized through comparison of select new data to the existing master database. This limits the need to recreate a large dataset for any change (however major or minor) to the process or raw material condition. A second benefit, related to the first, is that the cost barrier for a new supplier is greatly reduced. Equivalency testing allows smaller material producers to qualify their product relatively quickly and affordably and thus enables a larger supply chain. A similar approach to data handling will be required if additive manufacturing is to advance and mature into widespread use. Likewise, computationally driven material design in the aerospace market will not realize its full potential without a more adaptable approach to qualification testing and data handling.

\section{Unconstrained Potential}

Computationally driven materials design has already claimed some significant, well known successes in other market segments. The virtual aluminum casting program at Ford Motor Company is one such success [9]. The high-fidelity model neatly tying together the processmicrostructure-property relationship in aluminum engine block castings demonstrates the fundamental goal of the computational approach. This approach is well suited for processes such as additive manufacturing. In additive manufacturing, the controllable mass addition and thermal path offer the prospect of customizable structures with variable microstructures, chemistries, and properties. Predictive modeling can improve and accelerate advances already being made resulting in advances in alloy performance and the development of new classes of alloys. Demonstrations of gradient compositions, gas-phase in-situ alloying, functional density gradients, and other novel constructs have already been demonstrated by additive manufacturing techniques $[10,11,12]$. It is likely that the pace of adoption and integration of these new materials will be severely constrained in the aerospace market by the inability to fund a largescale data allowables program.

Likewise, incorporating processing advancements brought on through advanced thermal modeling techniques will also be limited due to the constraining nature of the qualification procedures. Improved thermal management strategies resulting in less distortion/residual stress are very desirable for optimizing the net shape capability of the process. Control of phase transformations in order to control microstructural morphology and scale are also very desirable. These and other advancements will be made available through the use of computational methods applied toward additive processes. The community of users, however, must be ready to accept these changes and find a better, more adaptable way of validating their outcomes. Otherwise, each new improvement becomes a new "process" requiring another expensive requalification of the material.

Finally, machine-to-machine variability can also add constraints to the overall maturation of the process. A dataset generated on a certain platform requires consistency not only from part-topart but also from machine-to-machine. This challenge is compounded by the equipment manufacturers constantly evolving hardware configurations. As the process models mature and begin to dictate the optimized operating conditions, the hardware will be required to adapt to these changes. This can only happen through a reformed data allowables procedure, one focused on the outcome of the process and not on the process itself. 


\section{Conclusions}

Aerospace structural metallic materials require a rigorous, expensive, and time consuming qualification procedure prior to their implementation onto an air vehicle system. This requirement creates a buffer that limits how quickly (if at all) promising new materials get introduced and fully adopted. The changing landscape of metallic material manufacturing creates a strong need for a fresh approach to qualification. The users with a vested interest must be willing to share precompetitive data in order to advance the broad industry. This is absolutely necessary for additive manufacturing to gain traction and expand beyond the few players fortunate enough to find a program willing to subsidize the huge cost of qualification.

Similarly, the computational materials engineering community also needs a better approach to data qualification. The promise of robust, validated modeling as a means to move away from the empirically dominated current approach will never come true without significant qualification reform. The shift away from a process-specified approach towards an outcome-based approach will be necessary in order to take full advantage of benefits new manufacturing methods have to offer. The combination of additive manufacturing with computationally driven materials design holds tremendous promise to create revolutionary new materials. Consideration for how these new materials get into the marketplace must become a priority.

\section{Acknowledgements}

I would like to thank my former colleagues at Lockheed Martin Aeronautic Company for their many years of insightful knowledge and guidance into the complicated world of air vehicle manufacturing. I would also like to thank my current colleagues at the NASA Langley Research Center for their support and insightful contributions to this paper.

\section{References}

1. J. Jackson, "Definition of Design Allowables for Aerospace Metallic Materials", (Paper presented at 2007 AeroMat Conference and Exposition, Baltimore, MD, 2007.

2. W.E. Frazier, D. Polakovics, and W. Koegel, "Qualifying of Metallic Materials and Structures for Aerospace Applications”, JOM, 53, 3 (2001), 16-18.

3. Metallic Materials Properties Development and Standardization (MMPDS-01), U.S. Department of Transportation, 2003.

4. J.T. Staley and W.H. Hunt, Jr., "Needs of the Aircraft Industry for Aluminum Products", (Paper presented at the $12^{\text {th }}$ Annual National Center for Manufacturing Sciences Technology Conference and Exposition, Orlando, FL, 1998).

5. "Accelerating Technology Transition: Bridging the Valley of Death for Materials and Processes in Defense Systems”, National Research Council, National Academies Press, Washington, D.C., 2004. 
6. Defense Advanced Research Projects Agency, Accelerated Insertion of Materials website: http://www.darpa.mil/dso/archives/aim/index.htm

7. C.A. Brice, S.D. Needler, and B.T. Rosenberger, "Direct Manufacturing at Lockheed Martin Aeronautics Company", (Paper presented at 2010 AeroMat Conference and Exposition, Bellevue, WA, 2010).

8. J.S. Tomblin, J.D. Tauriello, and S.P. Doyle, “A Composite Material Qualification Method that Results in Cost, Time, and Risk Reduction”, Proceedings of the $32^{\text {nd }}$ International SAMPE Technical Conference, Boston, MA, 2000.

9. J. Allison, M. Li, C. Wolverton, and X. Su, "Virtual Aluminum Castings: An Industrial Application of ICME”, JOM, 58, 11, (2006) 28-35.

10. R. Banerjee, P.C. Collins, D. Bhattacharyya, S. Banerjee, and H.L. Fraser, "Microstructural Evolution in Laser Deposited Compositionally Graded a/b Titanium-Vanadium Alloys", Acta Materialia, 51, 11, (2003) 3277-3292.

11. C.A. Brice, "Nitride Strengthened Titanium via Deposition Processing", Proceedings of the $11^{\text {th }}$ World Conference on Titanium, Kyoto, Japan, 2007.

12. B. Carcel, A.C. Carcel, I. Perez, E. Fernandez, A. Barreda, J. Sampedro, and J.A. Ramos, "Manufacture of Metal Foam Layers by Laser Metal Deposition", Proceedings of XVII International Symposium on Gas Flow, Chemical Lasers, and High-Power Lasers, Lisbon, Portugal, 2008. 